\title{
Face Recognition Algorithm Using Two Dimensional Locality Preserving Projection in Discrete Wavelet Domain
}

\author{
Geng Li ${ }^{1, *}$, Bing Zhou ${ }^{2}$, Yu-na Su ${ }^{1}$ \\ ${ }^{1}$ School of Computer Science and Technology, Henan Polytechnic University, Jiaozuo, China; ${ }^{2}$ School of Computer \\ Engineering, Jiaozuo University, Jiaozuo, China
}

\begin{abstract}
This paper proposes a new method for face recognition using Two Dimensional Locality Preserving Projection (2DLPP) in Discrete Wavelet Domain (DWT). Firstly, the dimensionality of the original face image is reduced by using the discrete wavelets transform and low frequency channel (LL) is selected to be as the features of face image. And then 2DLPP is applied to decrease the dimension of LL. Finally, the nearest neighbour classifier is used for face classification. The experiment results on ORL face database demonstrate the effectiveness of the proposed method.
\end{abstract}

Keywords: Face Recognition, 2DLPP, DWT.

\section{INTRODUCTION}

Face recognition is one of the most active research areas in computer vision and pattern recognition with practical applications that include forensic identification, access control and human computer interface and so on. Many face recognition methods have been developed, such as Principle Component Analysis (PCA), Linear Discriminant Analysis (LDA), etc. But a limitation of PCA and LDA is that they assume samples are subjected to multivariate normal distribution. Some researchers have shown that the face images are likely to be located in a low dimensional nonlinear manifold, may not obey the normal distribution, in this case, the PCA and LDA methods may fail. Locality Preserving Projection (LPP) is proposed for face recognition termed as Laplacian Faces recently and can be viewed as a linear version of Laplacian Eigenmap. Compared with PCA and LDA, LPP can preserve the local structure of the face images, whose performance is equivalent to LDA and far better than PCA [1-2].

However, an intrinsic limitation of classical LPP is that it involves the eigen decomposition, which is time-consuming especially for the high-dimensional face image data. In essence, an image matrix must be transformed into a vector, which may destroy the structural information residing in original two dimensional images. In order to overcome this limitation, 2DLPP is put forward. Its principal idea is to compute the covariance matrix based on two dimensional original [3] training image matrices, and to obtain its optimal projection matrix iteratively $[4,5]$.

The discrete wavelet transform (DWT) is a linear, invertible and fast orthogonal operation. As a feature extraction method, DWT has been used in various studies on face recognition, which can gain better recognition performance with less computational time. Also 2DPCA is used in DWT domain for face recognition. The motivation of this paper is to use 2DLPP in DWT domain with an aim to produce better face recognition performance with lower computational cost [6].

The remainder of this paper is organized as follows: in section 2 and section 3, we first give a review of 2DDWT and 2DLPP. Section 4 introduces the proposed method using 2DLPP in DWT domain. In Section 5, some experiments on standard face databases are given to evaluate the performance of the proposed method. Finally, conclusions are drawn in Section 6.

\subsection{D-DWT}

Wavelet transform is a kind of popular digital image processing tools. Wavelet transform of one-dimensional discrete signal is the convolution between a one-dimensional signal and a group of high and low filter pass. Wavelet transform for each level of the input is the output of the upper level low pass filter. DWT can be mathematically given as follows:

$$
D W T_{x(n)}=\left\{\begin{array}{l}
a_{p, q}=\sum x(n) g_{p}\left(n-2^{p} q\right) \\
d_{p, q}=\sum x(n) h_{p}\left(n-2^{p} q\right)
\end{array}\right.
$$

The coefficient $\mathrm{dp} / \mathrm{q}$ gives the frequency component details to the wavelet function and ap,q gives approximation components of the image. The functions $\mathrm{h}(\mathrm{n})$ and $\mathrm{g}(\mathrm{n})$ gives the coefficients of high pass and low pass filters respectively. The parameters $\mathrm{p}$ and $\mathrm{q}$ refers to wavelet scale and translation factors.

The two-dimensional discrete wavelet transform (2DDWT) is the extension of one dimensional discrete wavelet transform. When 2D-DWT is applied on an image, the image is divided into four sub-band images: LL (low frequency part of both horizontal and vertical direction), LH (low frequency part of horizontal direction, high frequency part of vertical 
direction), HL (high frequency part of horizontal direction, low frequency part of vertical direction) and $\mathrm{HH}$ (high frequency part of both horizontal and vertical direction). LL represents the main body of the image, whereas others represent the details of the image. Because the size of each subband image is half of original image, 2D-DWT can decrease the dimension of the image. Therefore, the dimension of the image can be furthermore reduced by applying 2D-DWT on LL part. Fig. (1). shows the main process of two level 2DDWT. Fig. (2) shows the result of one level 2D-DWT for an image selected from ORL face database [7-9].

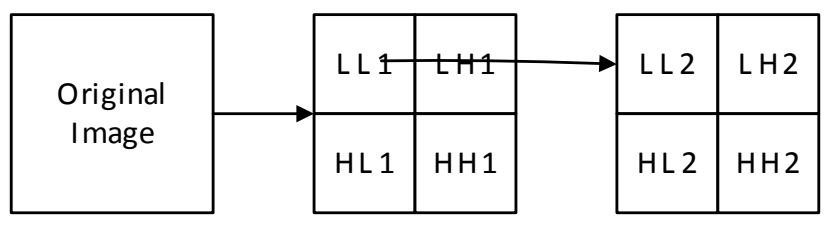

Fig. (1). Tow level 2D-DWT.
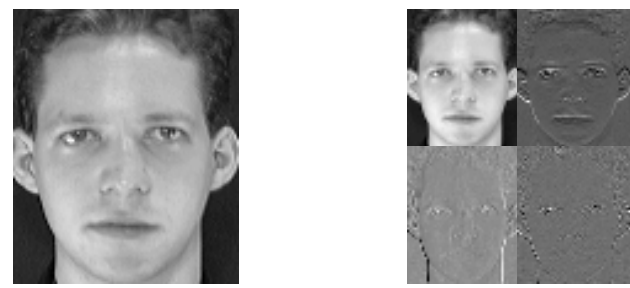

Fig. (2). One level 2D-DWT for an image from ORL.

From the Fig. (2), it can be seen that the LL sub band corresponds to low frequency components of an image and others are high frequency components of and image [10-11].

\subsection{DLPP}

LPP is an effective dimensional reduction method for face recognition. Contrary to conventional LPP, 2DLPP is based on $2 \mathrm{D}$ matrices rather than $1 \mathrm{D}$ vector. In order to describe how to use 2DLPP in frequency domain, now we first present 2DLPP briefly in spatial domain.

Let $x_{i} \in \mathfrak{R}^{m \times n}(i=1,2, \cdots, N)$ be the $N$ matrices of face images, we aim to compute two matrices $U \in \mathfrak{R}^{m \times r}$ and $V \in \Re^{n \times c}$ with orthonormal columns such that the projected low dimensional matrices preserve locality property. The low dimension feature is $y_{i}$ a $r \times c$ dimensional matrix given by $y_{i}=U^{T} x_{i} V$. Two projection matrices for the following objective function can be obtained by solving an optimization problem. The objective function of 2DLPP is defined as:

$\min \sum_{i, j}\left\|y_{i}-y_{i}\right\|^{2} W_{i, j}=\min \sum_{i, j}\left\|U^{T} x_{i} V-U^{T} x_{j} V\right\|^{2} W_{i, j}$

Where $W$ is a symmetry similarity measure matrix. A possible way of defining such $W$ is:

$W_{i, j}=\left\{\begin{array}{l}\exp \left(-\left\|x_{i}-x_{j}\right\|^{2} / 2 \sigma^{2}\right) \\ x_{i} \in N\left(x_{j}\right) \text { or } x_{j} \in N\left(x_{i}\right) \\ 0 \quad \text { others }\end{array}\right.$
Where $\sigma$ is an experience value, $N\left(x_{i}\right)$ is the set of its $k$ nearest neighbors of $x_{i}$. The value of $W_{i j}$ reflects the local information of samples, which depends on whether $x_{i}$ and $x_{j}$ are adjacent.

Let $D$ be a diagonal matrix, $D_{i i}=\sum_{j} W_{i j}$. The matrices $U$ and $V$ can be obtained by two steps as following:

(1) Fix $U$, then $V$ can be obtained by solving the following generalized eigenvector problem:

$\left(D_{U}-S_{U}\right) v=\lambda D_{U} v$

where

$D_{U}=\sum_{i} D_{i i} x_{i}^{T} U U^{T} x_{i}$

and

$S_{U}=\sum_{i} W_{i i} x_{i}^{T} U U^{T} x_{i}$

(2) Once $V$ is obtained, $U$ can be updated by solving the following generalized eigenvector problem:

$\left(D_{V}-S_{V}\right) v=\lambda D_{V} v$

where

$D_{V}=\sum_{i} D_{i i} x_{i} V V^{T} x_{i}^{T}$

and

$S_{V}=\sum_{i} W_{i i} x_{i} V V^{T} x_{i}^{T}$.

Thus, the optimal $U$ and $V$ can be obtained by iteratively computing the generalized eigenvectors in Equation (4) and (5). In our experiments, $U$ is initially set to the identity matrix [11].

\section{Proposed 2D-DWT and 2DLPP Approach}

In this section, we propose a new method based on 2DDWT and 2DLPP for face recognition. The block diagram of our method is shown in Fig. (3). The method involves two phases: training phrase and recognition phrase.

In training phrase, for each training face image, the DWT is applied to obtain its corresponding LL part (low frequency sub band) firstly. DWT can be furthermore applied on LL to reduce the size of the original image. The levels of DWT are determined by the fact that the ultimate LL can sufficiently represent the original image. Then 2DLPP are applied to ultimate LL to obtain optimal projection matrixes $U, V$ and recognition features of each training face image.

In recognition phrase, for each testing face image, the DWT is applied to obtain its corresponding LL part. The level of DWT is the same as one in training phrase. Then we can obtain recognition features by optimal projection matrixes $U$ and $V$. Finally, the nearest neighbor classifier is used to classify each testing face image.

\section{EXPERIMENTS}

In this section, we compare the performance of different four methods(2DLPP without using DWT, 1 level DWT/2DLPP, 2 level DWT/2DLPP and 3 level DWT/2DLPP) on ORL face database. 


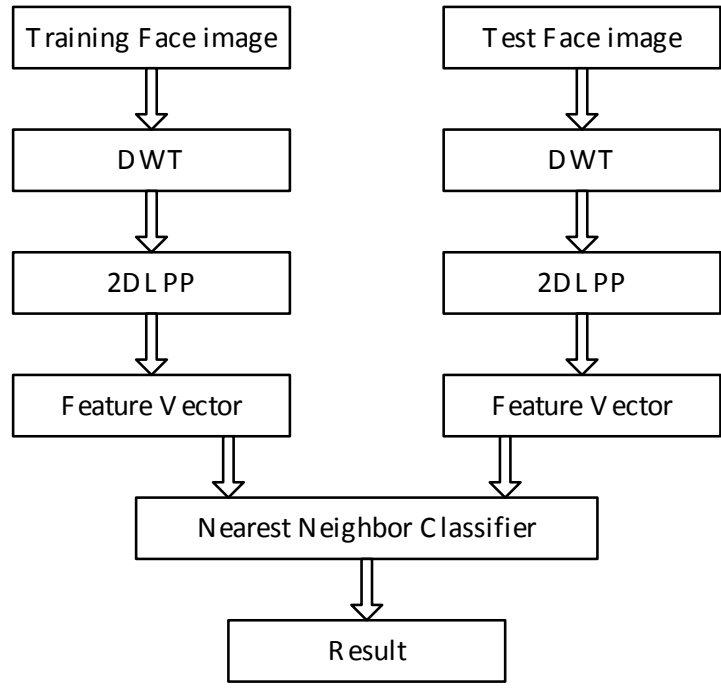

Fig. (3). The block diagram of proposed method.

The ORL database contains 40 persons, each having 10 different images. The images of the same person are taken at different times, under slightly varying lighting conditions and with various facial expressions. Some people are captured with or without glasses. The images in the database are manually cropped and rescaled to $112 \times 92$. Fig. (4) shows 10 sample images and corresponding diagonal images of one person in ORL.

In the experiments, we split the whole database into two parts evenly. One part is used for training and the other part is for testing. In order to make full use of the available data, we randomly select 3 images from each class to construct the training data set, the remaining images being used as the test images. In general, the recognition rates vary with the dimension of the face subspace. Table $\mathbf{1}$ shows the experimental results with first level DWT/2DLPP. Fig. 5(a) and (b) plot the corresponding eigvalue of the optimal $U$ and $V$ sorted by ascend. Fig. 5(c) plots the recognition rate when using different feature number of low-dimensional features.

When using 1 level DWT, the size of the original image with $112 * 92$ is reduced to $56 * 46$. Table 2 shows the experimental results with first level DWT/2DLPP. Fig. 6(a) and (b) plot the corresponding eigvalue of the optimal $U$ and $V$ sorted by ascend. Fig. 6(c) plots the recognition rate when using different feature number of low-dimensional features.
When using 2 level DWT, the size of the original image with $112 * 92$ is reduced to $28 * 23$. Table 3 shows the experimental results with second level DWT/2DLPP. Fig. 7 (a) and (b) plot the corresponding eigvalue of the optimal $U$ and $V$ sorted by ascend. Fig. 7(c) plots the recognition rate when using different feature number of low-dimensional features.

When using 3 level DWT, the size of the original image with $112 * 92$ is reduced to $14 * 12$.Table 4 shows the experimental results with first level DWT/2DLPP. Fig. 8(a) and (b) plot the corresponding eigvalue of the optimal $U$ and $V$ sorted by ascend. Fig. 8(c) plots the recognition rate when using different feature number of low-dimensional features.

From Figs. 5-8, we can see that the maximum of 2DLPP without DWT is $90 \%$. However, the maximum of 2DLPP with 1 level DWT, 2 level DWT and 3 level DWT are increased to $90 \%, 95.36 \%, 96.43 \%, 91.07 \%$ respectively. In addition, from Table 1-4, we can also see that the test time of 2DLPP with DWT is reduced when using the same dimension for recognition. So, we can conclude that 2DLPP with DWT can obtain better results with lesser time than 2DLPP without DWT.

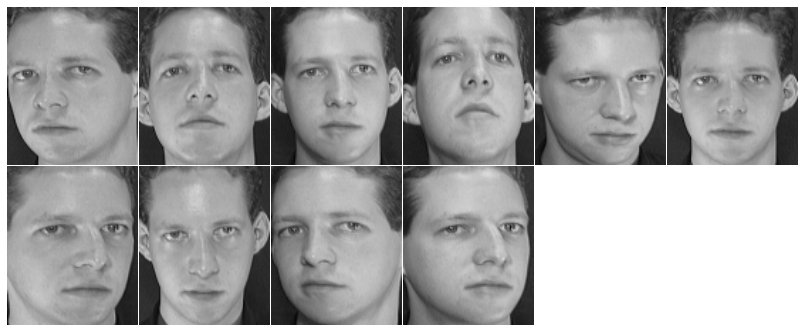

Fig. (4). Face images of one person in ORL.

\section{CONCLUSION}

In this paper, we propose a new method for face recognition using Two Dimensional Locality Preserving Projection (2DLPP) in Discrete Wavelet Domain. In fact, when applying DWT, main body information is stored in low frequency channel (LL) and some redundant information hidden in the original image can be reduced. So, the computational complexity is significantly reduced. The experimental results on ORL face database show that the recognition rate using 2DLPP in DWT domain is always better than its counterpart in spatial domain. At the same time, the test time is also reduced significantly.

Table 1. The result of experiment on ORL database using 2DLPP.

\begin{tabular}{|c|c|c|c|}
\hline \multirow{2}{*}{ Method } & Dimension & Recognition Rate & Test time(second) \\
\hline \hline \multirow{3}{*}{2 DLPP(112*92) } & $4 * 4$ & $89.29 \%$ & 10.75 \\
\cline { 2 - 4 } & $8 * 8$ & $87.5 \%$ & 10.90 \\
\cline { 2 - 4 } & $16 * 16$ & $86.43 \%$ & 10.81 \\
\cline { 2 - 4 } & $32 * 32$ & $86.07 \%$ & 11.71 \\
\cline { 2 - 4 } & $64 * 64$ & $84.29 \%$ & 14.82 \\
\hline
\end{tabular}




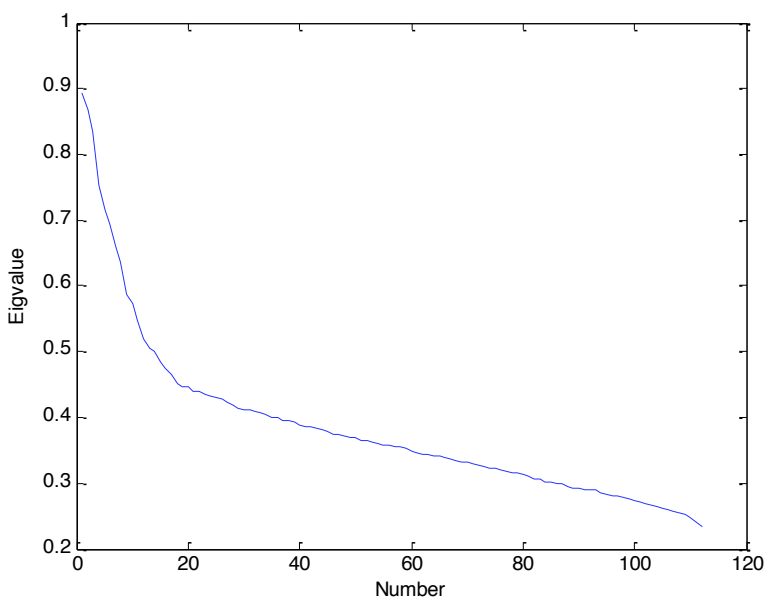

(a) Eigvalue of the optimal matrix $U$

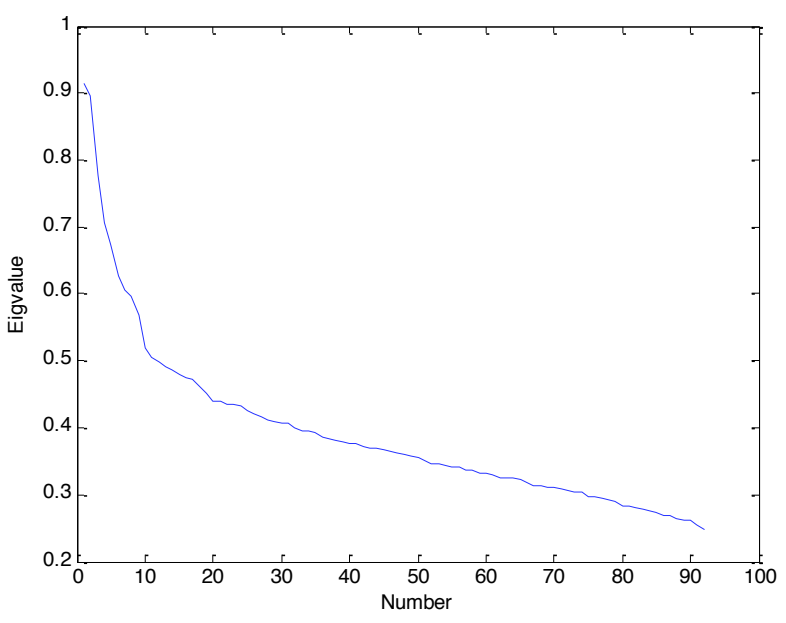

(b) Eigvalue of the optimal matrix $V$

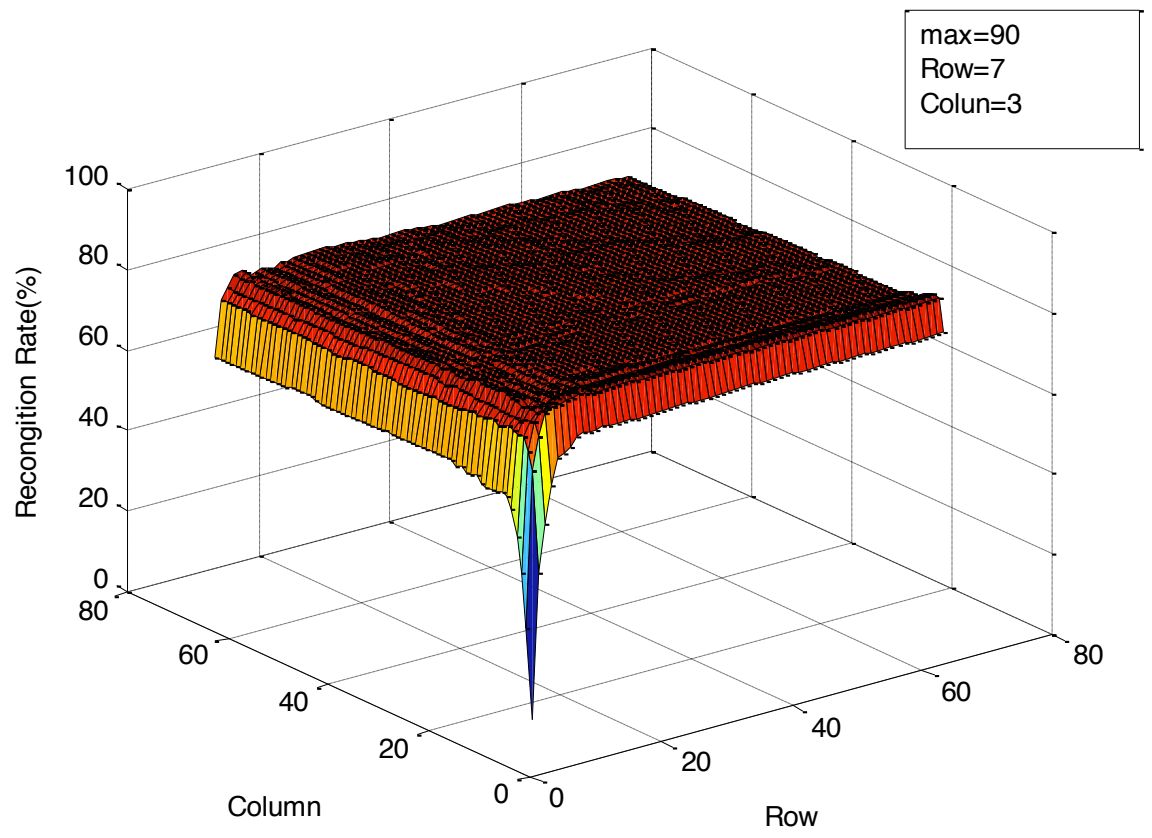

(c) Recognition accuracy of 2DLPP versus numbers of features

Fig. (5). The experimental result of 2DLPP on ORL database.

Table 2. The result of experiment on ORL database using 1-level DWT/2DLPP.

\begin{tabular}{|c|c|c|c|}
\hline \multirow{2}{*}{ Method } & Dimension & Recognition Rate & Test time(second) \\
\hline \hline \multirow{3}{*}{1 1-level DWT/2DLPP (56*46) } & $4 * 4$ & $91.43 \%$ & 1.74 \\
\cline { 2 - 4 } & $8 * 8$ & $93.57 \%$ & 1.77 \\
\cline { 2 - 4 } & $16 * 16$ & $93.57 \%$ & 1.83 \\
\cline { 2 - 4 } & $32 * 32$ & $93.57 \%$ & 2.41 \\
\hline
\end{tabular}




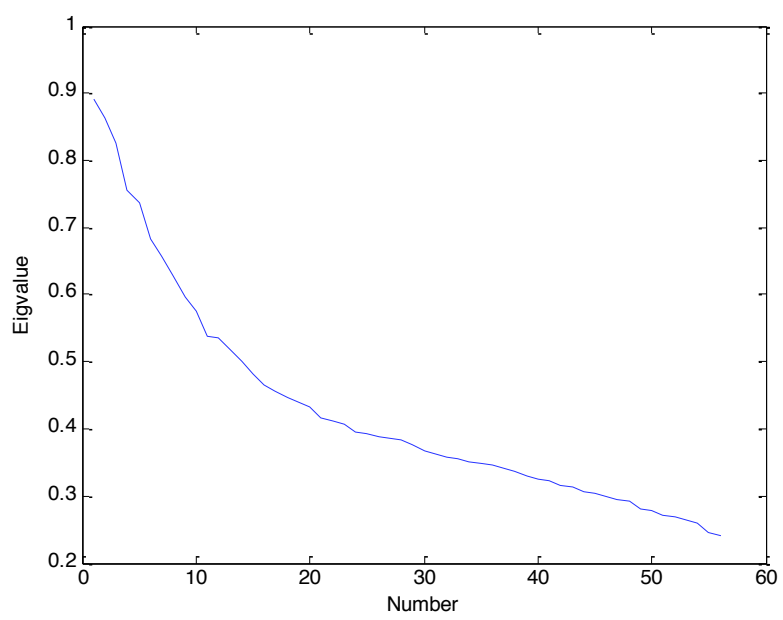

(a) Eigvalue of the optimal matrix $U$

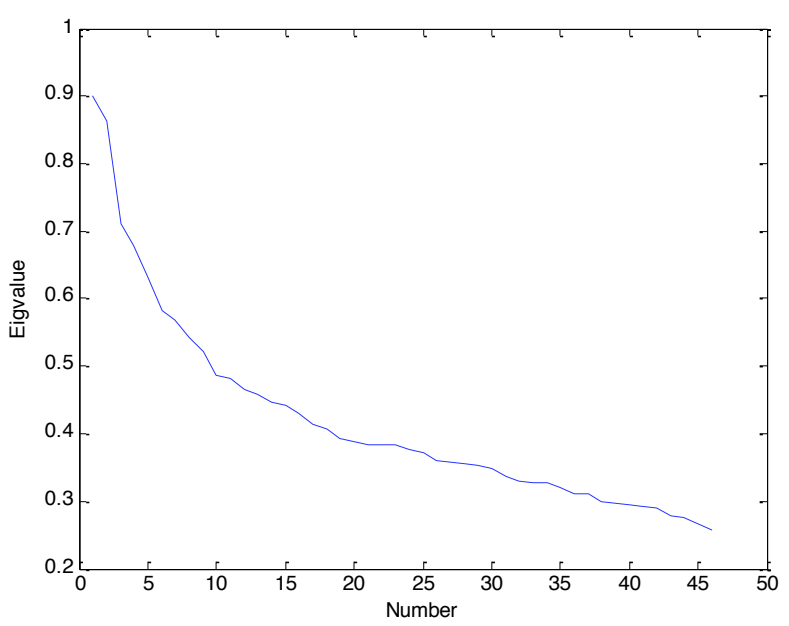

(b) Eigvalue of the optimal matrix $V$

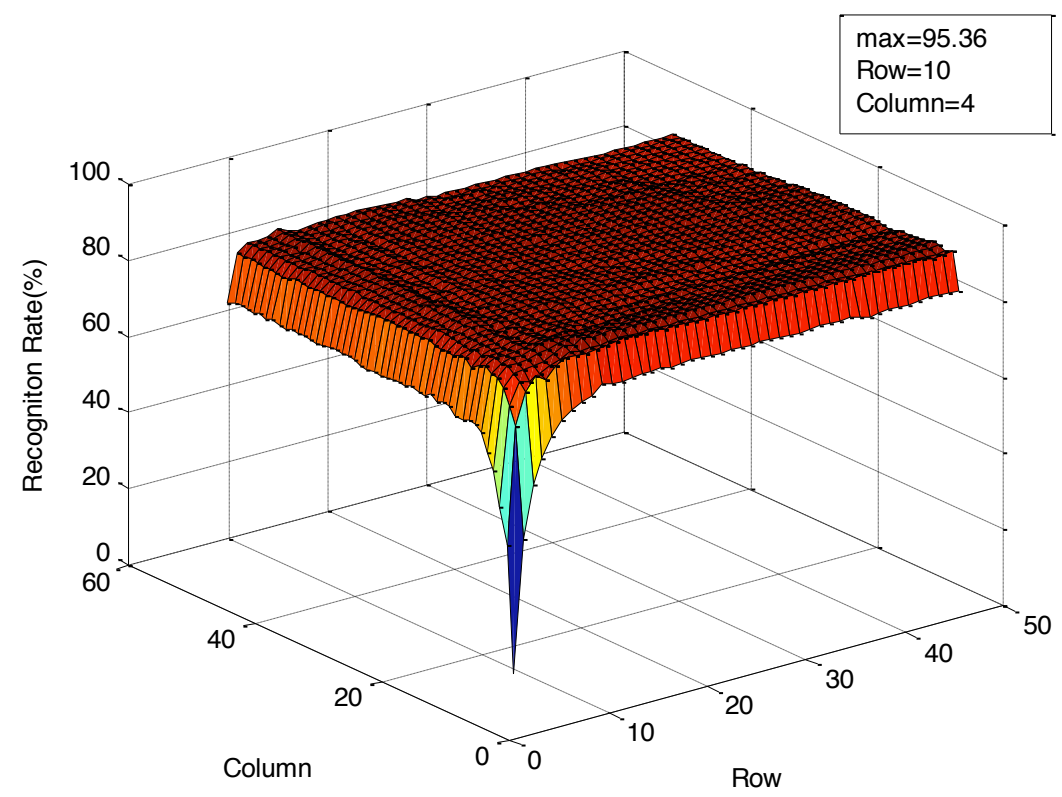

(c) Recognition accuracy of 1-level DWT/2DLPP versus numbers of features

Fig. (6). The experimental result of 1-level DWT/2DLPP on ORL database.

Table 3. The result of experiment on ORL database using 2-level DWT/2DLPP.

\begin{tabular}{|c|c|c|c|}
\hline \multirow{2}{*}{ Method } & Dimension & Recognition Rate & Test time(second) \\
\hline \hline \multirow{3}{*}{$2-$ level DWT/2DLPP (28*23) } & $4 * 4$ & $88.57 \%$ & 0.31 \\
\cline { 2 - 4 } & $8 * 8$ & $93.57 \%$ & 0.32 \\
\cline { 2 - 4 } & $16 * 16$ & $92.86 \%$ & 0.36 \\
\cline { 2 - 4 } & $23 * 23$ & 90.71 & 0.43 \\
\hline
\end{tabular}




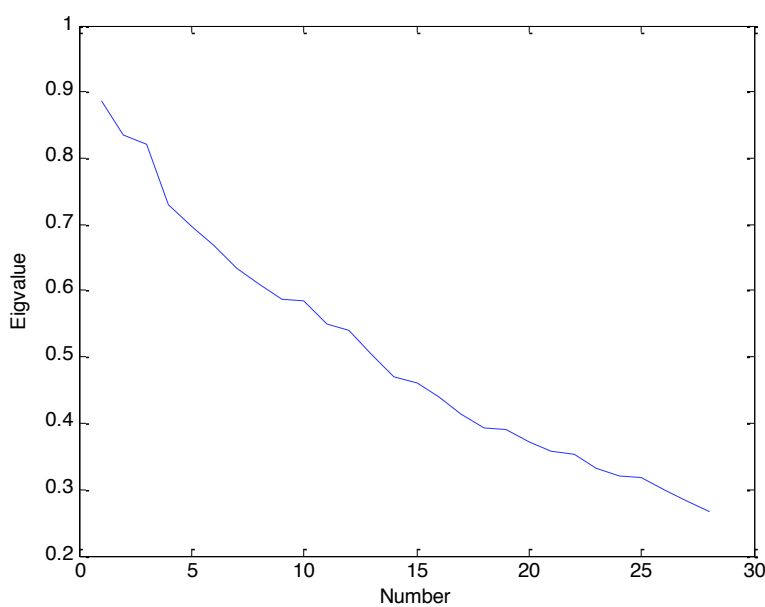

(a) Eigvalue of the optimal matrix $U$

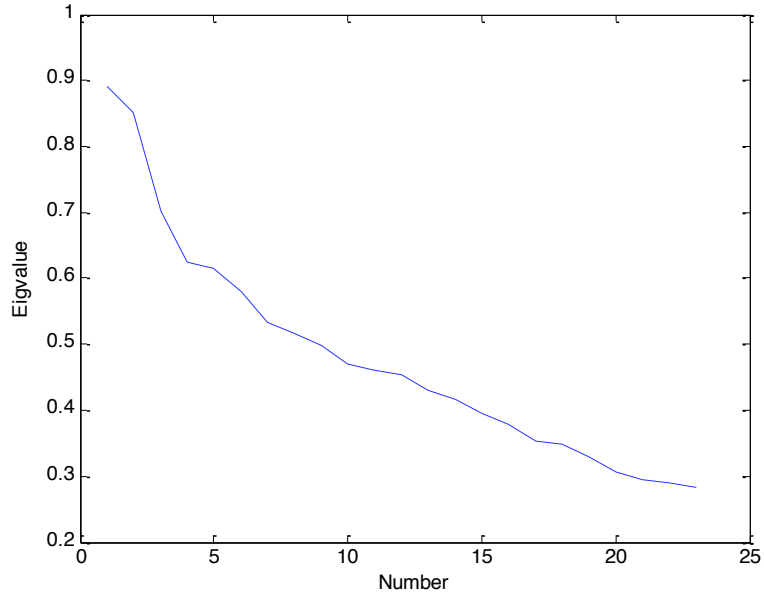

(b) Eigvalue of the optimal matrix $V$

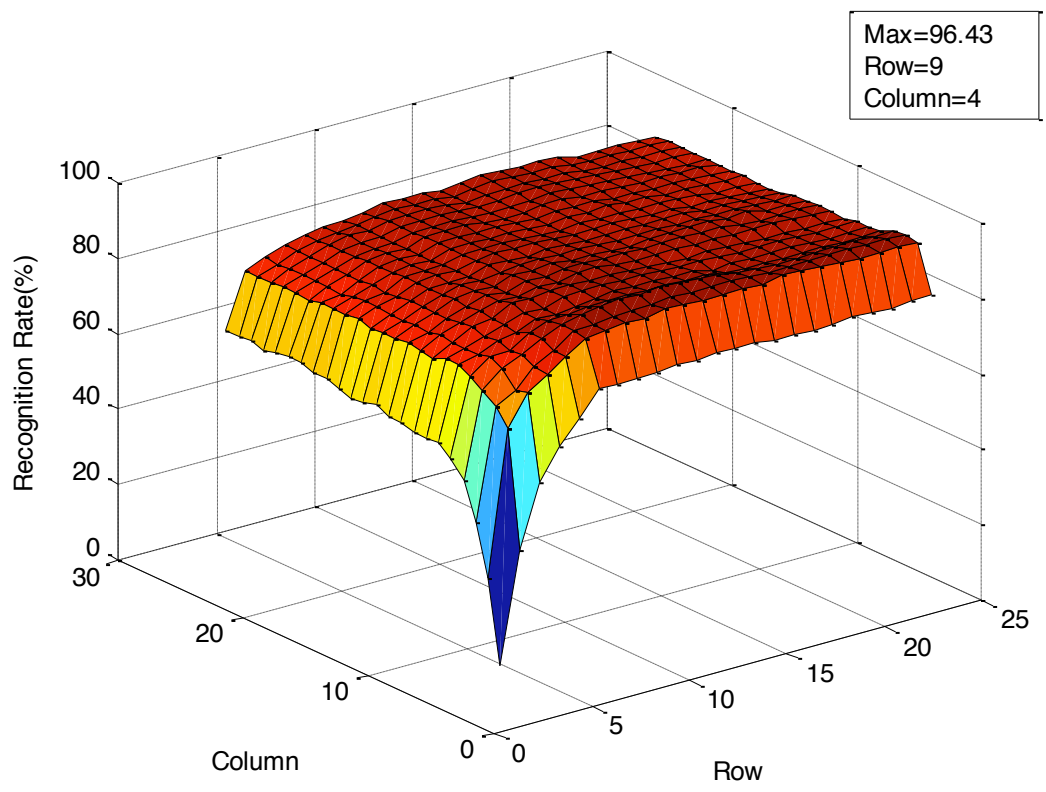

(c) Recognition accuracy of 2-level DWT/2DLPP versus numbers of features

Fig. (7). The experimental result of 2-level DWT/2DLPP on ORL database.

Table 4. The result of experiment on ORL database using 3-level DWT/2DLPP.

\begin{tabular}{|c|c|c|c|}
\hline \multirow{2}{*}{ Method } & Dimension & Recognition Rate & Test time(second) \\
\hline \hline \multirow{3}{*}{3 3-level DWT/2DLPP (14*12) } & $4 * 4$ & $87.5 \%$ & 0.09 \\
\cline { 2 - 4 } & $8 * 8$ & $87.5 \%$ & 0.10 \\
\cline { 2 - 4 } & $12 * 12$ & $88.57 \%$ & 0.12 \\
\hline
\end{tabular}




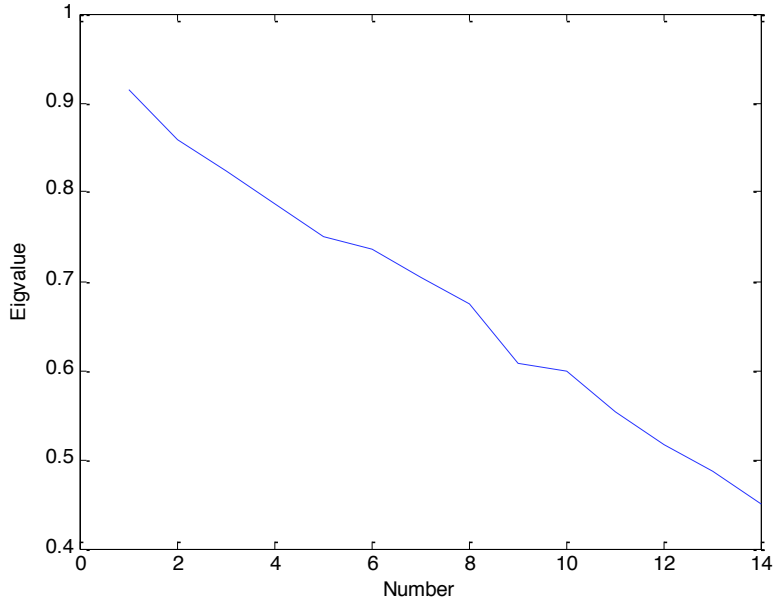

(a) Eigvalue of the optimal matrix $U$

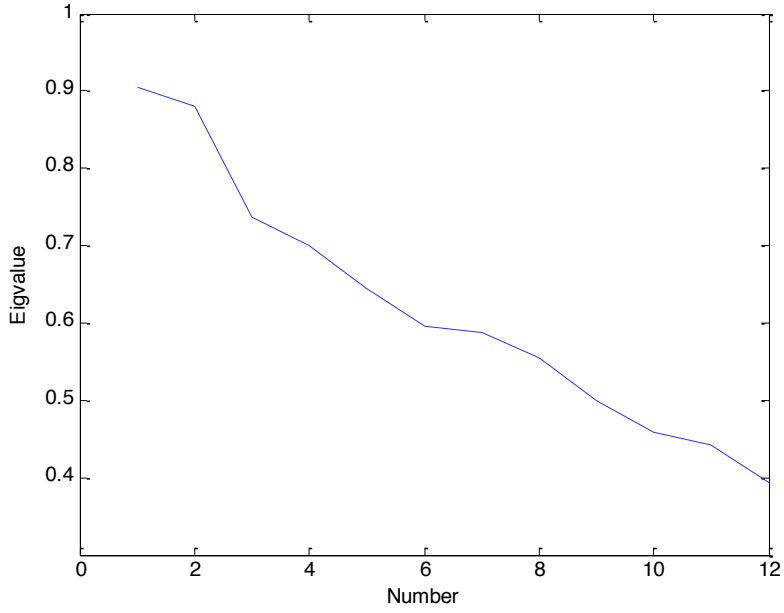

(b) Eigvalue of the optimal matrix $V$

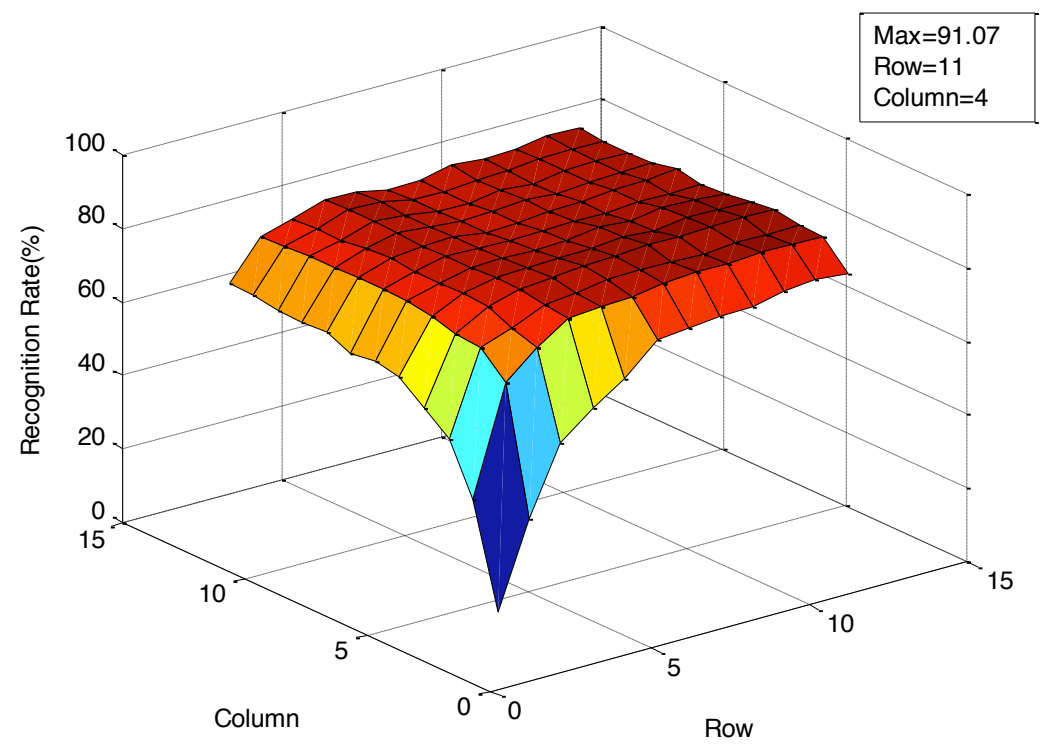

(c) Recognition accuracy of 3-level DWT/2DLPP versus numbers of features

Fig. (8). The experimental result of 3-level DWT/2DLPP on ORL database.

\section{CONFLICT OF INTEREST}

The authors confirm that this article content has no conflict of interest.

\section{ACKNOWLEDGEMENTS}

This work is supported by the Key Project of Guangxi Social Sciences, China (No. gxsk201424), the Education Science fund of the Education Department of Guangxi, China (No.2014JGA268), and Guangxi Office for Education Sciences Planning, China (No. 2013C108).

\section{REFERENCES}

[1] A. M. Martinez, A. C. Kak, "PCA versus LDA,"IEEE Trans. Pattern Analysis and Machine Intelligence, vol. 23, no. 2, 228-233, 2001.
[2] Belkin M, Niyogi P, "Laplacian eigenmaps for dimensionality reduction and data representation,"Neural Computation, vol. 15, no. 6, pp. 1373-1396, 2003.

[3] C.Lu, X. Liu, and W. Liu, "Face recognition based on two dimensional locality preserving projections in frequency domain ," $\mathrm{Neu}$ rocomputing, vol. 98, no. 12, pp. 136-142, 2012.

[4] X. F. He, and P. Niyogi, "Locality preserving projections ," Advances in Neural Information Processing Systems, Vancouver, British Columbia, Canada: 153-160, 2003.

[5] L. Zhu, Shan-an Zhu, "Face recognition based on two dimensional locality preserving projections," Journal of Image and Graphics, vol. 25 , no. 12, pp. 2043-2047, 2007.

[6] C. J. Liu, "Gabor feature based classification using the enhanced Fisher linear discriminant model for face recognition,". IEEE Trans on Image Processing, vol. 11, no. 4, pp. 467-476, 2000.

[7] Z. C .Liu, and Z. J. Fang, "PCA and FLD in DWT domain," Lecture Notes in Computer Science, vol. 24, no. 6, 197-204, 2012.

[8] M. Turk, and A. Pentland, "Eigenfaces for recognition," Journal of Cognitive Neuroscience, vol. 3, no. 1, pp. 72-86, 1991. 
[9] M. Belkin, and P. Niyogi, "Laplacian eigenmaps for dimensionality reduction and data representation", Neural Computation, vol. 15, no. 6, 1373-1396, 2003.

[10] U. I. Bajwa, I. A. Taj, and Z. E. Bhatti, "A comprehensive comparative performance analysis of Laplacianfaces and Eigenfaces for face recognition," Imaging Science Journal, vol. 59, no. 1, pp. 3240, 2011.

[11] Y. M. Wang, and Y. K. Wang, "Diagonal fuzzy linear discriminant analysis for face recognition," Journal of Computational Information Systems, vol. 9, no. 22, pp. 9121-9129, 2013.

Received: May 26, 2015

Revised: July 14, 2015

Accepted: August 10,2015

(C) Li et al.; Licensee Bentham Open.

This is an open access article licensed under the terms of the (https://creativecommons.org/licenses/by/4.0/legalcode), which permits unrestricted, noncommercial use, distribution and reproduction in any medium, provided the work is properly cited. 